DOI 10.1007/s00167-005-0717-7

Giulia Ottaviani

Pietro Randelli

Maurizio A. Catagni

\title{
Segmental cement extraction system (SEG-CES) and the Ilizarov method in limb salvage procedure after total knee cemented prosthesis removal in a former osteosarcoma patient
}

\section{Knee Surg Sports Traumatol Arthrosc (2005) 13:557-563}

Owing to an unfortunate oversight, the last line of the address for
M.A. Catagni (Lecco, Italy) was missing in the printed version of the article. The complete address is given here.

The online version of the original article can be found at http://dx.doi.org/10.1007/ s00167-004-0575-8

G. Ottaviani $(\bowtie)$

Institute of Pathology, University of Milan, Via della Commenda, 19, 20122 Milan, Italy

E-mail: giulia.ottaviani@unimi.it

Tel.: + 39-02-50320822

Fax: + 39-02-50320823

P. Randelli

U.O. Orthopaedics II, "Istituto

Policlinico", San Donato, MI, Italy

M. A. Catagni

Orthopaedic Department, "Alessandro

Manzoni” Hospital, Lecco, Italy 